\title{
Efficacy of a Novel Antibody TLR3 Modulator in the Self-Treatment of Common Cold: The ESTUAR Trial
}

\author{
Andrey Martyushev-Poklad1*, Jacques Bruhwyler², Stéphane Heijmans ${ }^{3}$, Michel Thiry ${ }^{4}$ \\ ${ }^{1}$ CONTRAFLU PTE LTD., Singapore City, Singapore \\ ${ }^{2}$ ECSOR, Blanmont, Belgium \\ ${ }^{3}$ ResearchLink, Linkebeek, Belgium \\ ${ }^{4}$ Theranor, Trooz, Belgium \\ Email: "amp@contraflu.com
}

Received 27 October 2015; accepted 22 December 2015; published 25 December 2015

Copyright () 2015 by authors and Scientific Research Publishing Inc.

This work is licensed under the Creative Commons Attribution International License (CC BY).

http://creativecommons.org/licenses/by/4.0/

(c) (i) Open Access

\section{Abstract}

Context: Since the discovery of toll-like receptor 3 (TLR3), no specific tools have been developed to modulate its activity in upper respiratory tract viral infections (URTIs). Contaflu ${ }^{\text {TM }}$ (antibodies to TLR3 cytoplasmic fragment) is the first specific TLR3 modulator that showed efficacy in a mouse model of influenza. Objective: To evaluate the efficacy of Contaflu in URTI. Methods: A doubleblind randomized placebo-controlled trial in adults with self-reported URTI (the ESTUAR trial) was conducted in 2012/2013 in Belgium. Adult outpatients started a 7-day treatment course with oral tablets of Contaflu or placebo within $36 \mathrm{~h}$ after onset of at least one of 4 typical symptoms of URTI. Patients were examined twice by their general practitioners, on days 2-3 and 10-14 after start of treatment. The primary endpoint was the overall severity of URTI calculated as the sum of Wisconsin Upper Respiratory Symptom Survey (WURSS-21) scores over the follow-up. Independent Student's t test was used to compare the disease severity between groups. Results: A total of 243 patients were enrolled by 32 investigators (121 Contaflu, 122 placebo); $92 \%$ of cases matched ICD codes J00 or J06. Most patients had very mild $(41.8 \%)$ or mild $(18.2 \%)$ URTI symptoms. In the ITT cohort, neither primary nor secondary outcome measures (duration of URTI, day-to-day and overall functional impairments) showed statistically significant differences between groups. The rate of adverse events was similar in both groups. In patients with moderate to severe URTI symptoms, Contaflu tended to reduce the overall disease severity, daily symptoms, and to improve the functional state. Due to the small size of the corresponding subgroups, Contaflu efficacy on daily scores was statistically significant $(p<0.05)$ only 1,2 , and 5 days after start of treatment. Conclusion: Contaflu was ineffective in mild URTI and showed efficacy in moderate to severe URTI cases.

\footnotetext{
"Corresponding author.
} 


\section{Keywords}

\section{Common Cold, Treatment, Toll-Like Receptor 3, TLR3 Modulation, Randomized Clinical Trial}

\section{Introduction}

Influenza and influenza-like illness (ILI) are among the major causes of morbidity and loss of productivity across the globe. Annual incidence of ILI, according to the US and UK data, amounts to about 35 - 45 cases per 100 persons [1] [2].

Influenza and ILI represent the most important group of viral respiratory tract infections in terms of severity, threat of complications and economic burden. Annually direct influenza-related medical costs in the USA amount to $\$ 10$ billion, while overall economic losses amount to $\$ 87$ billion, or $0.8 \%$ of gross domestic product [3].

Over the last two decades considerable progress has been made in understanding the mechanisms underlying ILI severity, in particular, the role of pattern recognition receptors [4]-[6]. However, none of these important discoveries have translated so far into emergence of new effective and safe treatments for influenza or ILI [7] [8]. This problem is especially important bearing in mind that over the first 5 days after influenza virus invasion, innate response plays the leading role in antiviral protection [9]. Impaired regulation of body response to the infection (excessive production of inflammatory factors and insufficient efficacy of protective factors) is responsible for severe forms of influenza [9].

There are objective problems that impede development of novel therapeutics for common cold and ILI, including products targeting the components of innate host response to viruses.

1) Wide diversity of viruses causing common cold and ILI; variability of influenza virus (which contributes to the development of resistance to antivirals).

2) While most physiological functions require modulation in diseased condition, all generally accepted pharmacological tools affecting the immune system exploit one-directional effects: either inhibition or stimulation. There are no true immune modulators available in the largest markets, like EU and USA.

3) Many promising targets for therapeutic intervention are located inside the cell. Generally accepted pharmacological tools are based on binding the active substance with the target molecule. That is why in most cases it is impossible to simultaneously combine access of the active substance into the cell, specificity of binding to the target, and safety.

Most of the listed problems can be overcome with the use of an original drug development technology platform which can be identified as “Antibody-based Modulation of Biological Response” (AMBRe). This platform proposed by the first author is based on his prior experience of developing several antibody-based therapeutics for the Russian market. A detailed overview of the technology ancestor to AMBRe can be found in an online monograph [10].

Contaflu ${ }^{\mathrm{TM}}$, the first product developed with AMBRe platform, targets human toll-like receptor type 3 (TLR3) and is designed for the treatment of influenza and ILI.

The important role of TLR3 in the pathogenesis of severe forms of influenza is well documented [11]-[13].

During the preclinical development of Contaflu, several candidates were tested for efficacy in a mouse model of non-lethal influenza (Burleson Research Technologies, USA) [14]. Rabbit antibodies raised against the target of Contaflu, the FYW peptide, a 15-amino acid peptide sequence from cytoplasmic domain of human TLR3 (FYWNVSVHRVLGFKE, amino acids 732-746), appeared to produce the best therapeutic effect both on severity and duration of clinical influenza (unpublished data).

Since the active substance of Contaflu is purified antibodies specific to a small peptide sequence, the probability of cross-reactivity with other molecular targets in humans is nihil. This assumption is supported by the search for cross-reactivity in Uniprot (http://www.uniprot.org), the most extensive open database of proteins: no cross-reactivity is found between the FYW peptide and any other human protein. This high specificity of Contaflu molecular target implies that the product's therapeutic effects can only be attributed to specific action on TLR3 functioning, most probably, to modulation of TLR3 signaling.

In usual drug development, successful preclinical proof of efficacy is followed by preclinical safety studies, and if successful-by Phase I clinical trial (safety in healthy humans). But in the case of Contaflu, there were 
several sound reasons to proceed directly to Phase II clinical trials without additional tests for safety. These reasons formulated as Risk Assessment were found acceptable by Health Authorities that examined the Clinical Trial Application, namely, Belgian Ministry of Health (AFMPS/FAGG) and the Independent Ethics Committee (IEC) of the Free University of Brussels (Erasme Hospital).

The conclusion of Risk Assessment was the following: "Considering the absence of toxicity of rabbit immunoglobulin per se and the innocuous nature of the anti-FYW peptide affinity of immunoglobulin, considering also the fact that Contaflu is administered only via the oral route and that the immunoglobulins are diluted below one molecule per dose, animal toxicity studies on Contaflu were considered as unnecessary and unethical with respect to animal welfare."

Also, Contaflu solid dosage form meets the regulatory criteria established for first safe dilution, i.e. dilution which poses no risk to patients and for which submission of non-clinical safety assessment is unnecessary [15].

The objective of the first phase II clinical trial of Contaflu was the evaluation of the product's efficacy as an early treatment of the most prevalent self-limiting viral infection in humans, generally known as common cold (non-febrile acute respiratory infections-J00, Acute nasopharyngitis, and J06, Acute upper respiratory infections of multiple and unspecified sites).

Common cold is a general term for a clinical condition caused by a wide range of respiratory viruses, the most important of which is rhinovirus [16]. TLR3, the target of Contaflu, is known to be implicated in the pathogenesis of rhinovirus infection [17], hence it was hypothesized that Contaflu would reduce both disease severity and duration, and accelerate functional recovery in patients with common cold.

Primary objective: To evaluate the efficacy of Contaflu in reducing the severity of symptoms of common cold in otherwise healthy adults.

Secondary objectives:

- To evaluate the efficacy of Contaflu in reducing the duration of common cold.

- To evaluate the impact of Contaflu on health-related Quality of Life (functional impairment) in patients with common cold.

- To evaluate the safety of Contaflu in patients with common cold.

\section{Methods}

\subsection{Trial Design}

To reach our objectives, we organized a multicentre, randomized, double-blind, parallel, placebo-controlled study evaluating the efficacy and safety of Early Self-Treatment of viral Upper respiratory tract infections with homeopathic oral Antibodies to the TLR3 FYW peptide (Contaflu ${ }^{\mathrm{TM}}$, used under technical name TAO1), or ESTUAR study. There was a balanced allocation between Contaflu (TAO1) and placebo (1:1). No changes in methods were introduced after trial commencement.

The study protocol, any amendments, the informed consent and other information that required pre-approval were reviewed and approved by the IEC of ULB Erasme Hospital.

This study was conducted in accordance with "good clinical practice" (GCP) and all applicable regulatory requirements, including, where applicable, the Declaration of Helsinki.

\subsection{Participants}

Initial enrolment criteria: outpatients aged $\geq 18$ years, who were able and willing to adhere to the study protocol and signed the informed consent. To be actually enrolled in the study, the patients needed to have self-reported recently emerged symptoms of common cold (answer "Yes" to the question "Do you believe that you are coming down with a cold?”) and at least one of the following 4 common cold symptoms: sneezing, runny nose, nasal obstruction and/or sore throat, with no limitations of symptom severity.

Exclusion criteria for initial enrolment were conditions where immune reactivity might be impaired: specific chronic diseases (autoimmune disease, chronic bronchitis, human immunodeficiency virus (HIV) infection, lupus, rheumatoid arthritis), malignancy for which the patient had undergone resection, radiation or chemotherapy within the last five years, cancer therapy, immunosuppressant therapies, use of systemic corticosteroids, a history of asthma or allergic rhinitis and corresponding symptoms (itchy eyes, sneezing, wheezing). Exclusion criteria for enrolment in treatment protocol were any of the common cold symptoms persisting since more than 36 
hours, use of other homeopathic drugs designed to treat URTIs, use of antibiotics, anti-histaminergic drugs or decongestants, and participation in another clinical trial within one month prior to treatment start.

\subsection{Settings and Locations}

This study was conducted by thirty-one (31) principal investigators (General practitioners) and one (1) coordinating investigator (Dr. Stephane Heijmans, ResearchLink, Brussels) in one single country, Belgium. All investigators were members of the ResearchLink's network of investigators.

At Visit 1, initial enrolment, the medications, questionnaires and diary cards (DC) were dispensed to healthy individuals who had signed the informed consent. Upon contracting a common cold, they had to start the treatment immediately and to take contact with the doctor by phone within 36 hours. On Day 2-3 after the onset of disease, they had to visit the doctor (Visit 2) to confirm the diagnosis and validate the actual enrolment. The doctor had to check if questionnaires were filled in correctly. On the 10-14th day (at the latest) after start of treatment, Visit 3 was planned to pick up questionnaires, evaluate safety, disease complications and treatment compliance.

The treatments were taken by patients at home; visits took place in the physician's offices.

\subsection{Interventions}

Treatment group: Contaflu (TAO1) tablets (to be dissolved in the mouth, not to be swallowed; should be taken at least 10 minutes apart from meals and/or smoking):

Day 1: 3 tablets to be taken over the first 2 hours of treatment, then 3 tablets over the rest of Day 1.

Days 2-3: 5 tablets/day (intakes distributed evenly over the daytime).

Days 4-7: 3 tablets/day (intakes distributed evenly over the daytime).

Control: Placebo tablets: same dosage regimen as for Contaflu (TAO1).

Duration of treatment: 7 days.

Concomitant medications: Only oral analgesics/antipyretics, such as paracetamol or ibuprofen, were allowed in case of fever or headache. Each intake of such medications was to be registered in the patient's DC.

Contaflu (TAO1) active substance is a mixture of homeopathic dilutions of purified rabbit antibodies to a cytoplasmic peptide sequence of human toll-like receptor type 3 (anti-TLR3): FYWNVSVHRVLGFKE.

\subsection{Outcomes}

The ability of Contaflu to affect severity and duration of common cold was assessed with the use of a patient reported outcome instrument, The Wisconsin Upper Respiratory Symptom Survey (WURSS). WURSS was developed using individual interviews and focus groups among community-recruited people with Jackson-defined colds. It was designed to be an evaluative outcomes instrument, aimed at measuring change over time in patient-valued illness domains.

The Wisconsin Upper Respiratory Symptom Survey-21 (WURSS-21) is a validated tool to evaluate the duration and severity of common cold. It is constituted of twenty-one categorical questions (Likert-type 7-level severity scale) [18].

Patients were requested to complete this questionnaire daily, from Day 1 to Visit 3 (Day 10 to 14).

\subsubsection{Primary Efficacy Endpoint}

Severity of symptoms of common cold: Decrease in severity was assessed as reduction in average area under the curve (AUC) under the time severity curve (of the sum of WURSS scores 2-20 over the follow-up). A 30\% reduction in AUC versus placebo was considered as clinically significant.

\subsubsection{Secondary Efficacy Endpoints}

Duration of common cold: Duration was defined as the number of days from self-reported onset of disease to the last day before the participant answered "Not sick" to the question, "How sick do you feel today?" A 2-day reduction in average disease duration versus placebo was considered as clinically significant.

Functional impairments in the course of the disease:

- Day-to-day scores for functional impairments domain (questions 12-20). 
- Overall (sum of all WURSS scores related to functional impairments over the follow-up).

- The need of symptomatic analgesics/antipyretics (average number of intakes).

Evaluation of safety: adverse events (AEs) and serious adverse events (SAEs) coded using the Medical Dictionary for Regulatory Activities (MedDRA) assessed according to their frequency, severity, outcome and relationship to the study drug.

Data collection: Paper Case Report Form (CRF).

All efficacy and safety endpoints were assessed after completion of the follow-up period based on the data extracted from the CRFs. No changes were introduced to trial outcomes after trial initiation.

\subsection{Sample Size, Randomization, Blinding, and Statistical Methods}

The sample size had been estimated on the basis of the study of Barrett et al. [18] and reconfirmed by calculation performed at ECSOR using nQuery Advisor (Version 7.0).

A sample size of 115 in each group would have 80\% power to detect a difference in mean AUCs of 103 (a difference of $30 \%$ between a Placebo AUC mean of 310 and a Contaflu-TAO1 AUC mean of 207) assuming that the common standard deviation was 277 using a two group t-test with a 0.05 two-sided significance level.

Patient numbers were assigned sequentially to patients consenting to participate in the study.

The randomisation list was prepared for each Investigator by the Independent Biostatistician of ECSOR as the Contract Research Organization (CRO), using a block size of 2 with the software nQuery Advisor Version 7.0.

Packaging, labelling, storage and distribution of investigational medicinal products were performed by a pharmaceutical services company authorized by competent authorities, B\&C Group SA (Belgium). Based on the randomization list received from the $\mathrm{CRO}, \mathrm{B} \& \mathrm{C}$ Group labelled each package with individual patient identification code.

The product doses were distributed to the Investigators while respecting the randomisation block size. After having checked the eligibility criteria of the patient at Visit 1, the Investigator provided the treatment package corresponding to the patient identification code. A randomisation blocking scheme (1:1 ratio; block size = 2) was used to ensure that balance between treatments was maintained.

The Independent Biostatistician (CRO) and the Safety Officer were the only persons with permanent access to the randomisation codes.

Individual sealed envelopes containing randomisation codes were provided to each Investigator.

The study was double-blind. The patient, the Investigator, his/her staff and the sponsor ignored to which group the patient belonged. Contaflu (TAO1) and placebo tablets had exactly the same appearance (form, size, colour, aspect) and chemical composition.

The procedure of blinding was performed by B\&D Group in full compliance with applicable regulations.

The primary cohort for the efficacy and safety assessments was the intention to treat (ITT) population. It included patients having presented common cold symptoms and for whom at least one post-treatment information had been recorded in the CRF. The secondary cohort for the efficacy and safety assessments was the per protocol (PP) population. It included patients having completed the study, who did not deviate from the protocol and in whom compliance was at least $80 \%$ during the whole treatment period.

\subsubsection{Efficacy Analysis}

The mean AUC of common cold severity (sum of total scores of 19 questions of the WURSS-21 at all time points) was compared between the two groups using the Independent Student's t test. Mean duration of common cold was compared between the two groups using Mann-Whitney's test.

Functional impairments in the course of the disease:

Day-to-day mean scores for functional impairments domain was compared between the two groups using analysis of variance (ANOVA) for repeated measures with the factor "time", "treatment" and "time-treatment interaction" as classification criteria. Appropriate post-hoc test was used to compare the two groups at each time point if and only if the interaction of the ANOVA was significant $(\mathrm{p}<0.05)$.

Mean overall sum of all WURSS scores related to functional impairments over the follow-up was compared between the two groups using the Independent Student's t test.

Need of symptomatic analgesics/antipyretics (average number of intakes) was compared between the two groups using the Independent Student's t test. 


\subsubsection{Safety Analysis}

AEs and SAEs were analysed descriptively in terms of frequency, severity, outcome and relationship to the study drug. The overall rates of AEs and SAEs were compared between the two groups using Chi-square tests or Fisher's exact tests, as appropriate.

\subsubsection{Additional Data-Driven Analysis}

Analysis of the clinical course of common cold.

In patients where WURSS scores were evaluable for at least 5 successive days, we analyzed the patterns of common cold clinical course and the actual need in drug treatment.

Additional subgroup analysis was performed in patients of ITT cohort with moderate to severe common cold symptoms who had signs of systemic response to the infection upon inclusion (baseline WURSS score $\geq 38$ ), and in the subgroup of patients with mild/very mild baseline severity (WUSS score $<38$ ). For these subgroups, the mean AUC of common cold severity, mean daily and mean daily functional symptom scores were compared between the two groups using an Independent Student's t test.

In all statistical tests, the difference was considered significant if p value was below 0.05 .

\section{Results}

\subsection{Participant Flow}

The participant flow in the ESTUAR trial is presented in Figure 1.

Overall, 244 patients were enrolled into the study (Total cohort) by 32 investigators. Overall, 243 patients completed the study. For one patient (Patient 356), the informed consent collection process was not correctly conducted. The data of this patient cannot be used. Therefore the Intention-To-Treat (ITT) cohort included 243 patients (122 patients in the placebo group and 121 in the Contaflu-TAO1 group).

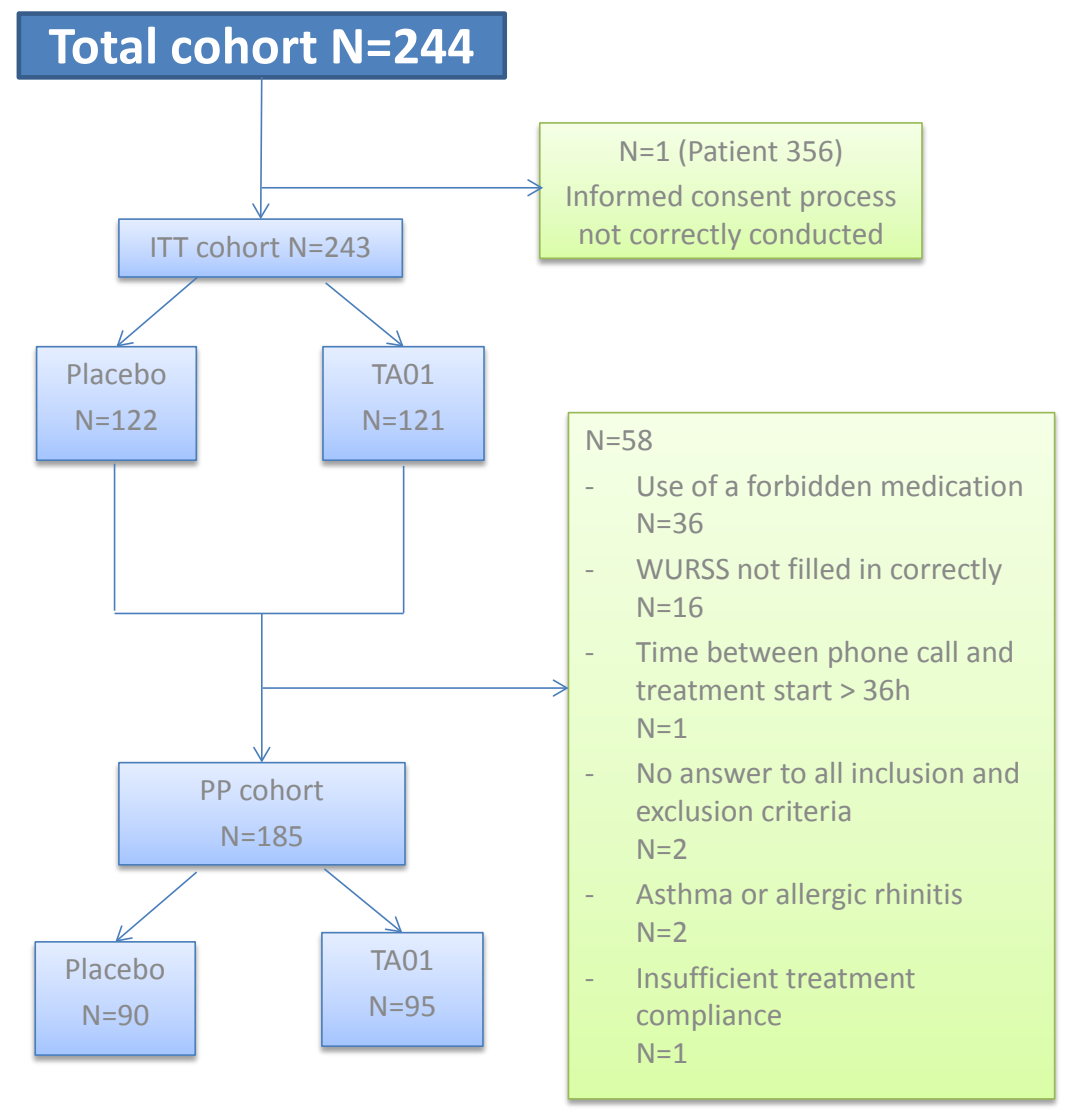

Figure 1. Participant flow in ESTUAR trial. 
Fifty-eight $(\mathrm{N}=58)$ patients were eliminated from the Per Protocol (PP) cohort:

- 36 used a forbidden medication.

- 16 did not fill in the WURSS correctly.

- 1 for whom the time between the phone call and the treatment start was longer than $36 \mathrm{~h}$.

- 2 for whom an answer was not provided for all inclusion and exclusion criteria.

- 2 had asthma or allergic rhinitis and.

- 1 had insufficient treatment compliance.

These were the main causes of elimination for each patient. It was frequent that several causes were present at the same time for one patient.

Overall, there were 185 patients in the PP cohort: 90 patients in the placebo group and 95 patients in the Contaflu-TAO1 group.

Natural history of an URTI (WURSS scores for at least 3 days after start of treatment) was evaluable for 113 patients in placebo and 111 patients in Contaflu-TAO1 group.

Subgroup analysis of URTI severity and duration for moderate to severe common cold covered 89 patients, 43 in placebo, and 46 in Contaflu-TAO1 group. Subgroup analysis for common cold of (very) mild baseline severity covered 89 patients, 45 in placebo, and 44 in Contaflu-TAO1 group.

The first patient was included on 17 July 2012. The last patient was included on 24 May 2013. The last patient last visit was on 16 July 2013. The trial was ended with reaching the pre-defined target sample size necessary for statistical analysis.

\subsection{Baseline Data}

Mean age, gender distribution and baseline common cold severity of the patients in the ITT cohort are given in Table 1. All but two patients were Caucasians (2 Hispanics). There were no statistically significant differences between groups: $\mathrm{p}=0.701$ for age and $\mathrm{p}=0.277$ for gender.

Most of patients (80.9\%) had Acute nasopharyngitis (J00), 11.2\% had Acute upper respiratory infections of multiple and unspecified sites (J06), 1.7\% had Influenza, virus not identified (J11.1), the rest had tracheitis/ bronchitis/coryza/sinusitis/tonsillitis.

There were no clinically or statistically ( $p=0.464$ in the ITT cohort; Mann-Whitney's tests) significant differences between the two groups in terms of severity of the common cold at baseline. This was assessed by the first question of the WURSS: How sick do you feel today?

There were no clinically or statistically significant ( $p=0.741$ in the ITT cohort; Mann-Whitney's tests) differences between the two groups in terms of functional score at baseline.

Analysis of treatment outcomes was performed in ITT cohorts based on evaluable outcomes: 114 patients for placebo, 117 patients for Contaflu, total 231 patients. For the rest 12 patients, only baseline WURSS scores were available.

\subsection{Primary Endpoint}

The primary endpoint was the decrease in common cold severity, assessed as a reduction in average area under the curve (AUC) under the time severity curve (of the sum of all 19 relevant WURSS scores over the follow-up period). A 30\% reduction in AUC versus placebo was to be regarded as clinically significant.

There were no clinically or statistically significant $(p=0.945$ in the ITT cohort and $p=0.490$ in the PP cohort using independent Student's t tests; $\mathrm{p}=0.999$ in the ITT cohort and $\mathrm{p}=0.357$ in the PP cohort using MannWhitney's tests) differences between the two groups for the primary endpoint (Table 2).

Table 1. Demographic and clinical characteristics.

\begin{tabular}{cccc}
\hline Characteristic & Placebo N = 122 & Contaflu-TAO1 N = 121 & All N = 243 \\
\hline Age, mean (SD) & $47.2(16.2)$ & $46.5(14.8)$ & $46.9(15.5)$ \\
Females, number (\%) & $77(63.1)$ & $85(70.2)$ & $162(66.7)$ \\
$\begin{array}{c}\text { Severity score of the common cold at } \\
\text { baseline, mean (SD) }\end{array}$ & $3.3(1.9)$ & $3.1(2.0)$ & $3.2(1.9)$
\end{tabular}


Table 2. Area under the curve under the time severity curve (sum of all WURSS scores except questions 1 and 21) (ITT cohort).

\begin{tabular}{ccccc}
\hline \multirow{2}{*}{ Randomization } & & AUC & & \\
\cline { 2 - 5 } & $\mathrm{N}$ & Mean & SD \\
\hline Placebo & 116 & 187.5 & 176.2 \\
Contaflu-TAO1 & 117 & 185.8 & 177.4 \\
\hline
\end{tabular}

\subsection{Secondary Endpoints}

\subsubsection{Common Cold Duration}

Duration of common cold was defined as the number of days from self-reported onset of disease to the last day before the participant answered "Not sick" to the question, "How sick do you feel today?" A 2-day reduction in average disease duration versus placebo was to be regarded as clinically significant.

There were no clinically or statistically significant ( $p=0.792$ in the ITT cohort; Mann-Whitney's tests) differences between the two groups in terms of common cold duration.

\subsubsection{Overall Functional Score over the Whole WURSS Recording Period}

There were no clinically or statistically significant $(\mathrm{p}=0.839$ in the ITT cohort using independent Student's $\mathrm{t}$ tests) differences between the two groups in terms of mean AUC under the time functional impairment curve (sum of WURSS functional scores 12-20) over the recorded follow-up (Table 3).

\subsubsection{Concomitant Medications}

Overall, 152 (62.6\%) patients of the ITT cohort took concomitant medications (76 in the placebo group and 76 in the TAO1 group).

\subsection{Ancillary Analyses}

\subsubsection{Analysis of the Natural History of Common Cold}

A detailed analysis of the clinical course of common cold (changes of WURSS Questions 2-20 summed score over time) in ESTUAR patients suggested that the severity trajectories vary much across the tested cohort. Surprisingly, 32 patients (13\% of ITT cohort) answered "Not sick" to WURSS question 1 "How sick do you feel today?” already on Day 1 meaning that with very mild local symptoms of common cold patients had no disease-related functional problems.

We also found that severity trajectory (judged from mean summed WURSS score) in the present study differed considerably from the data obtained in WURSS validation study ([18], Figure 1): in particular, median summed score for ESTUAR on Day 2 was 30.5, while in WURSS validation study it was about 50 (see Figure 2).

That is why for the sake of drawing a more accurate picture of the disease in ESTUAR study we attempted to divide the evaluable patient population (with evaluable WURSS scores for at least 5 successive days, a total of 224) into two groups:

1) Patients with mild or very mild course of the disease (baseline total WURSS score < 38) —a total of 134 patients (70 in placebo, 64 in Contaflu-TAO1 group);

2) Patients with moderate to severe clinical manifestations of the disease (baseline score $\geq 38$ ) -a total of 90 patients (43 in placebo, 47 in Contaflu-TAO1 group).

Obviously, the subgroup of patients with moderate to severe baseline symptoms matched well the patient population studied in the WURSS validation study [18].

The trajectories of common cold severity in the both groups (daily total WURSS scores and functional WURSS scores) are given in Figure 3.

The two groups differed considerably both in severity of clinical manifestations, functional impairments, disease duration and eventually in the necessity of drug treatment.

Patients with mild baseline symptoms achieved general alleviation of clinical symptoms (summed WURSS score $\leq 10$ ) three days earlier, and alleviation of functional impairments (summed WURSS functional score $\leq$ 5)—four days earlier than patients with moderate to severe baseline symptoms. 


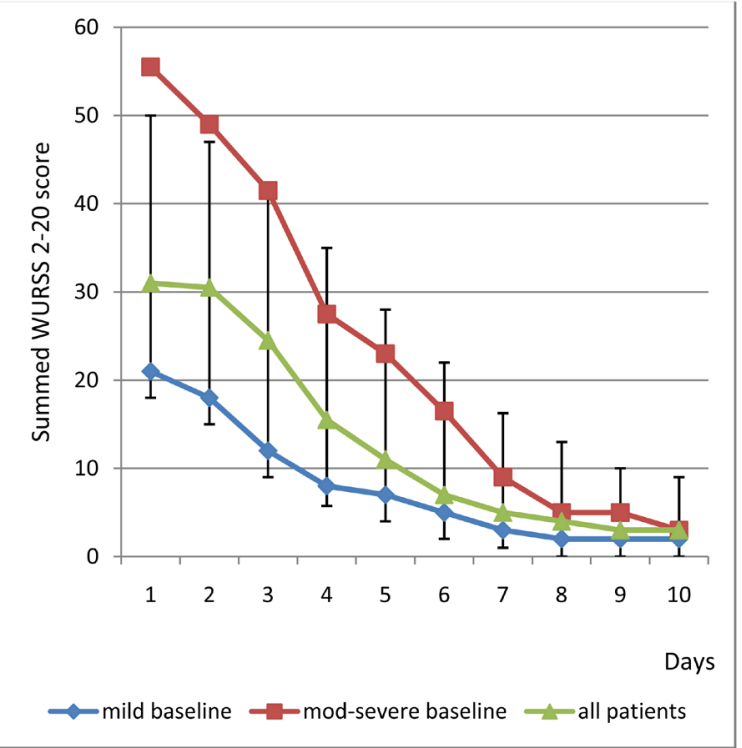

Figure 2. Trajectories of common cold severity (median summed WURSS 2-20 score) in the whole ITT cohort, in patients with mild and moderate to severe baseline clinical manifestations. (Median daily summed WURSS scores; the end of vertical lines indicate $25 \%$ and $75 \%$ percentiles for whole ITT cohort).

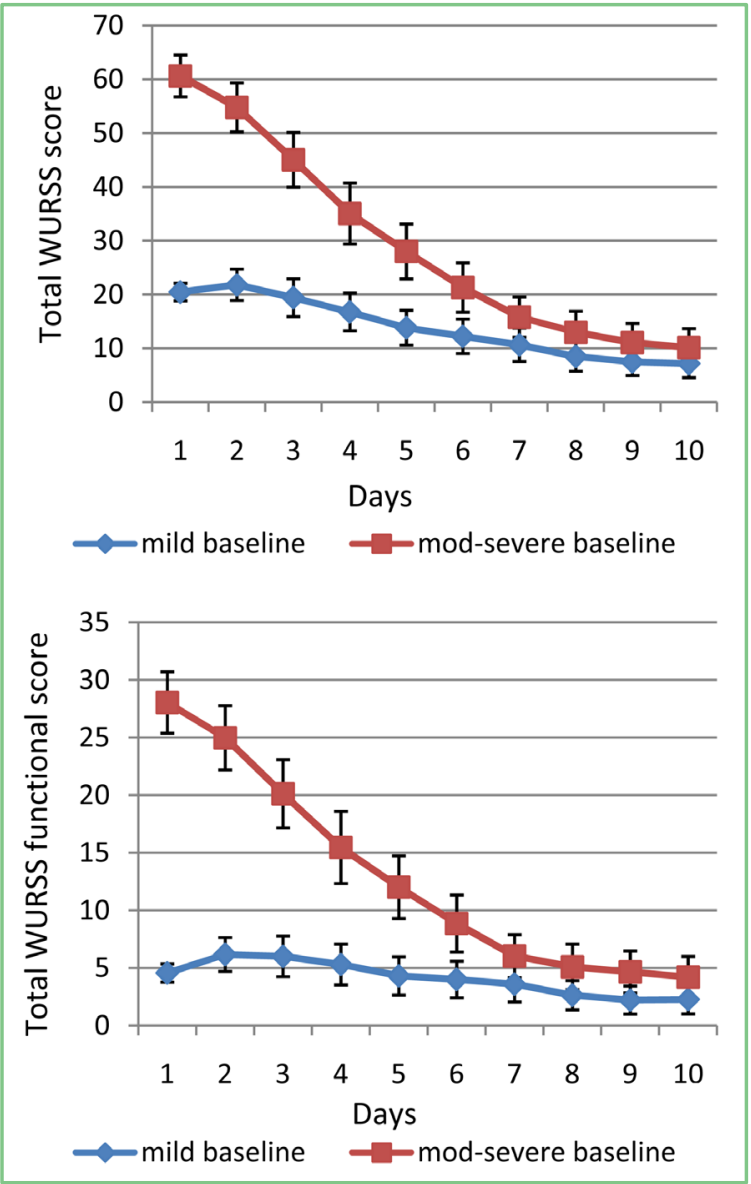

Figure 3. Trajectories of disease severity and functional impairments in patients with mild and moderate to severe baseline WURSS (2-20) symptom scores (mean $\pm 95 \%$ confidence interval). 
Table 3. Secondary endpoints (ITT cohort).

\begin{tabular}{ccccccc}
\hline \multirow{2}{*}{ Randomization } & \multicolumn{3}{c}{ Common cold duration (days) } & \multicolumn{2}{c}{ AUC under the time functional impairment curve } \\
\cline { 2 - 7 } & $\mathrm{N}$ & Mean & SD & N & Mean & SD \\
\hline Placebo & 92 & 5.2 & 3.1 & 116 & 79.0 & 95.1 \\
Contaflu-TAO1 & 84 & 5.4 & 3.3 & 117 & 76.5 & 89.3 \\
\hline
\end{tabular}

Symptomatic NSAIDs (paracetamol/ibuprofen) were used in 13.4\% (18 out of 134) patients with mild baseline symptoms and 32.2\% (29 out of 90) patients with moderate to severe symptoms - 2.4 times more often.

\subsubsection{Daily WURSS Scores in Contaflu-TA01 versus Placebo Patients with Moderate to Severe Baseline Symptoms}

Since the patients of these two subgroups had very distinct severity trajectories of common cold, we further evaluated the efficacy of Contaflu-TAO1 in patients with moderate to severe symptoms-those who had considerable functional impairments and need in drug therapy (Figure 4 and Figure 5).

There was no statistically significant difference in baseline scores between these Contaflu-TAO1 and placebo subgroups.

Patients of Contaflu-TAO1 group $(n=46)$ demonstrated faster improvement of both overall symptom score and functional score compared to placebo group $(n=43)$.

The use of Contaflu tended to reduce total disease severity (by 22\%), with reduction of daily symptoms by $20 \%$ $40 \%$, and improvement of functional state by up to $45 \%$ on day 5 after start of treatment. Due to small size of the corresponding subgroups, Contaflu efficacy on daily WURSS scores reached statistically significant level ( $\mathrm{p}<$ 0.05 ) only 1,2 , and 5 days after start of treatment.

Interestingly, patients with mild baseline symptoms receiving Contaflu-TAO1 $(n=64)$ had more pronounced clinical symptoms and later recovery than their placebo counterparts $(n=70)$. The difference in daily functional scores was statistically significant on days 3-5 and 8-10 of the follow-up.

\subsection{Safety Results}

\section{Adverse Events (AEs)}

Overall, 198 AEs were reported by 91 patients (37.4\%) in the ITT cohort: 88 AEs were reported by 46 placebo patients and $110 \mathrm{AEs}$ were reported by 45 Contaflu-TAO1 patients. All but one AE were considered non-related to the study medication by the investigator.

It should be noted that the vast majority of reported AEs were directly linked to the disease itself and represented symptoms of viral URTIs. This is the case for fatigue, fever, asthenia, general body pain, muscle pain, headache, coughing, voice loss, nasal congestion, runny nose and sneezing. All the other symptoms are not uncommon in viral URTIs; even gastrointestinal symptoms can be caused by some viruses known to be the cause of URTIs. Certain symptoms may simply be regarded as complications of the main disease (otitis media, sinusitis, bronchitis).

There was no statistically significant difference in the rate of AEs between the two study groups $(\mathrm{p}=1.000$; two-sided Fisher's exact test).

Overall conclusion drawn by the investigators: Contaflu-TAO1 can be considered as a safe treatment.

\section{Discussion}

In ESTUAR trial we attempted to evaluate the clinical efficacy of Contaflu ${ }^{\mathrm{TM}}$, a novel medicinal product that presumably produces its therapeutic effect through modulation of TLR3-related host response. Although preclinical proof of efficacy had been performed in an animal model of influenza, we chose another medical condition for the first in human trial-non-influenza URTI (common cold), the most prevalent form of URTI.

The study was designed in the manner to reproduce most closely a real life situation of self-treatment of common cold: the medication was distributed to healthy volunteers, common cold was self-diagnosed, and the decision about start of treatment was made early by the patient him/herself.

We selected WURSS21, a tool for measuring patient reported outcome (PRO), as the most relevant instrument 


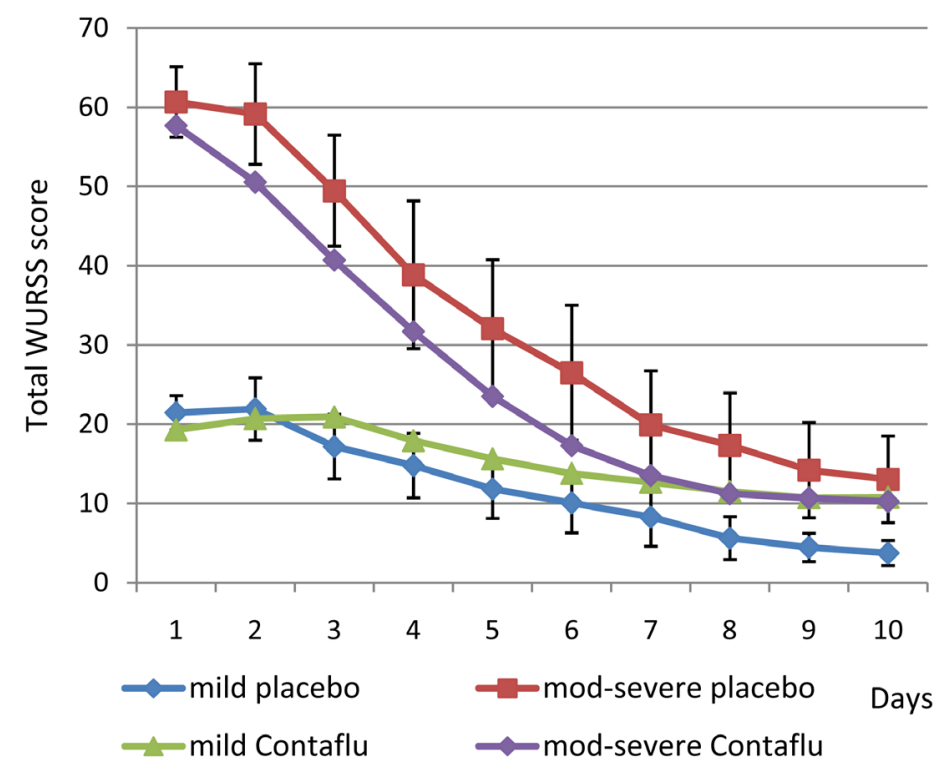

Figure 4. Daily summed WURSS (2-20) scores in patients with different baseline severity (mean $\pm 95 \%$ confidence interval).

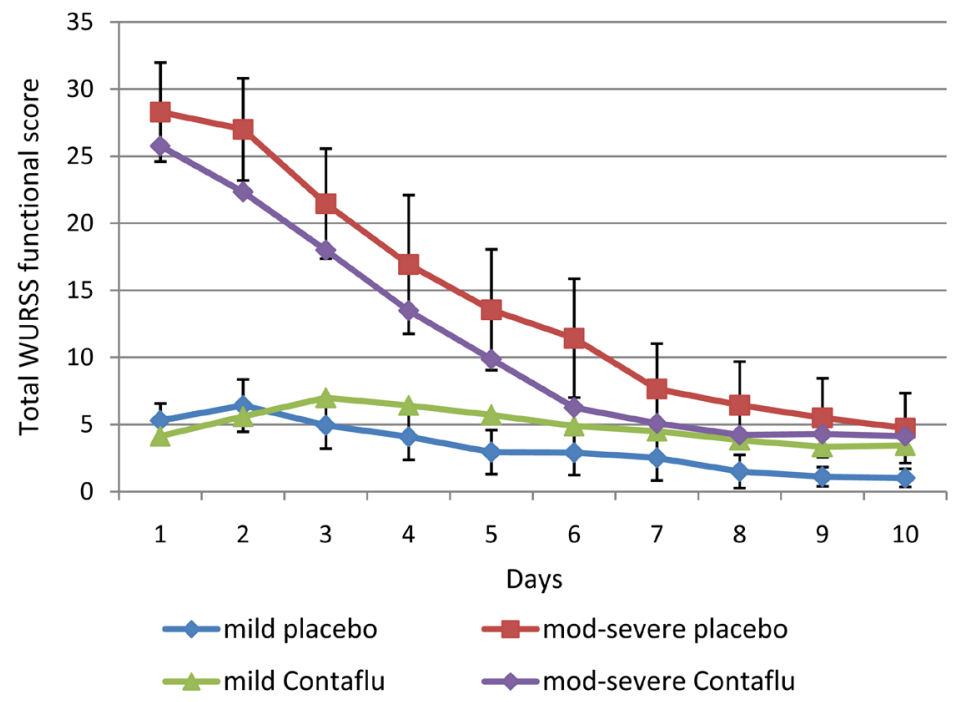

Figure 5. Daily summed WURSS functional (12-20) scores in patients with different baseline severity (mean $\pm 95 \%$ confidence interval).

since there is a growing awareness that PRO are more adequate in patient-centered care solutions [19].

Selection of such a design had a certain drawback: the trial happened to involve a large share $(60 \%, 134$ out of 224) of patients with mild and very mild forms of common cold. Most of these patients had only minimal functional impairments, recovered very quickly, and actually didn't need any drug treatment at all.

Due to prevalence of mild forms of common cold in the studied patient population, the disease severity trajectory in ESTUAR patients differed much from the patients involved in the benchmark study focused on validation of WURSS questionnaire [18].

A possible conclusion that can be drawn from this finding is that in real-life common colds about $50 \%$ of patients have mild clinical manifestations that don't affect daily functioning and resolve within 4 - 5 days without treatment. These patients usually do without contacting a doctor. In this patient population Contaflu-TAO1 was useless or even could have prolonged disease duration. 
A separate analysis performed in the subgroup of ESTUAR patients matching the benchmark WURSS validation study showed that Contaflu-TAO1 tended to reduce both overall disease severity and duration, as well as improve functioning of the patients. However, due to small sample size $(n=90)$ these effects failed to reach the level of statistical significance.

Positive evidence of Contaflu efficacy in moderate to severe human URTI, very often presented with systemic symptoms, agree well with preclinical findings, where the product was effective in mouse influenza of moderate severity accompanied by systemic clinical signs.

The fact that Contaflu alleviated disease symptoms in moderate and severe cases while producing a moderate aggravation of the symptoms in mild cases of common cold supports the hypothesis that it modulates TLR3 activity. TLR3 is known as one of the few key molecules responsible both for induction of adaptive response essential for virus elimination-on the one hand, and mediating pathogenesis of exacerbated antiviral response (hence severity of clinical manifestations)-on the other hand. In other words, "TLR3 participates in both defense and offence in host immunity to viruses” [20]. That is, adequate antiviral response requires a certain optimal level of TLR3 functioning. Since Contaflu enhanced clinical signs of local inflammatory response in mild common cold and alleviated systemic and local symptoms in moderate to severe cases, the utility of this medication should be limited to URTI with pronounced inflammation, especially with systemic symptoms.

\subsection{Limitations}

A major part (60\%) of patients involved in ESTUAR trial had mild or very mild clinical manifestations of URTI, therefore the overall conclusion about non-superiority of Contaflu-TAO1 over placebo cannot directly apply to moderate to severe forms of URTI. This is supported by results of subgroup analysis. But the actual efficacy of Contaflu in the latter conditions has to be confirmed in a separate controlled trial focusing either on moderate to severe URTI cases, or influenza and influenza-like illnesses.

An important note on the source of potential bias has to be made with respect to the instrument used to measure efficacy endpoints in different URTIs.

The impact of systemic and local (respiratory) symptoms on both overall disease severity and functional impairment has cardinal differences in common cold compared to influenza. According to the authors of patient-reported outcome instrument designed for influenza, FluiiQ questionnaire [21], functional impairments in influenza are mostly caused by organism's systemic response to the infection, while the impact of respiratory symptoms both on functional and emotional state is minor. Therefore, only less than $30 \%$ of functional impairments in influenza are determined by local respiratory symptoms.

Unlike influenza, in most common cold cases systemic symptoms are absent or very mild; hence the functional state becomes much more influenced by local respiratory symptoms. Thus, in colds the role of systemic symptoms in overall disease severity assessed by WURSS21 and in the impact on functional state, ranges from 0 (if systemic symptoms are absent) to maximum of 20\% (2 of 8 questions on physical symptoms of the disease). That is why a medicinal product affecting the local mechanisms of the infection will show efficacy in common cold if evaluated with the use of WURSS21.

A product affecting mostly systemic mechanisms of the disease may fail to show efficacy in common cold due to two reasons: 1) systemic symptoms are absent in mild cases of common cold; 2) the very structure of the questionnaire tends to mask the effects of a product affecting primarily systemic symptoms of the infection.

Therefore, a proper choice of the questionnaire is very important for adequate measurement of the product's efficacy. For Contaflu, which is presumably more active against systemic symptoms of URTI, WURSS may appear to be not the best efficacy evaluation instrument.

\subsection{Generalizability of the Trial Findings}

The major finding of ESTUAR-lack of Contaflu efficacy in mild cases of common cold-can be considered as reliable, and can be well extrapolated to other populations, such as patients below 18 years old. The evidence of Contaflu efficacy in moderate to severe cases of URTI and influenza has to be further confirmed in a separate controlled clinical trial, with the use of another questionnaire (like FluiiQ) reflecting more adequately the role of systemic symptoms in perception of drug efficacy.

Patient population in ESTUAR trial involved individuals with concomitant chronic conditions which may serve as contraindications for some symptomatic medications for URTI: gastric ulcer (2 patients), arterial 
hypertension (21 patients), and diabetes (3 patients). Although Contaflu was safe in these and other adult patients, and although current regulations consider it safe due to the nature of its active substance, the product's safety in specific chronic diseases has to be further backed by controlled clinical trials.

\section{Conclusions}

The ESTUAR trial has proven safety but failed to demonstrate clinical efficacy of Contaflu ${ }^{\mathrm{TM}}$-TAO1 in selftreatment of URTI due to the following reasons:

1) (Most important) Incorrect selection of inclusion criteria: as a result, $60 \%$ patients had very mild common cold that basically didn't need treatment and where Contaflu was ineffective. The effect in patient population where Contaflu was effective had therefore been insufficient to reach statistical power.

2) Other features of study design: low weight of systemic symptoms in the overall WURSS score; inclusion of Day 1 score (before treatment) in the measurement of overall severity and scores for days after the disease had generally resolved (i.e. all symptoms were very mild).

All these weaknesses in trial design will be corrected in the next clinical trial. This is a common situation for the first exploratory phase 2 trials of novel investigational products.

\section{Authors' Contributions}

AMP and MT are co-inventors of Contaflu ${ }^{\mathrm{TM}}$-TAO1; AMP contributed to trial design and analysis of results, and wrote the major part of the paper; JB (ECSOR) provided all statistical analysis and calculations, managed the flow of information throughout the study, and was responsible for Study Report; SH (Research Link) contributed to study design, was responsible for all clinical aspects of the study, and coordinated the team of investigators; MT coordinated all organizational aspects of the study, and contributed to the article preparation.

\section{Other Information}

Trial Registration: The ESTUAR trial is registered at EUDRACT \#2012-000057-31 and the US National Institutes of Health (ClinicalTrials.gov) \# NCT01651715. Funding: Funding for ESTUAR was provided by Theranor sprl (Belgium), the owner of patent rights on Contaflu ${ }^{\mathrm{TM}}$.

\section{References}

[1] Benson, V. and Marano, M.A. (1998) Current Estimates from the National Health Interview Survey, 1995. National Center for Health Statistics. Vital Health Stat 10 (199). http://www.cdc.gov/nchs/data/series/sr_10/10_199_1.pdf

[2] Adler, A.J., Eames, K.T., Funk, S. and Edmunds, W.J. (2014) Incidence and Risk Factors for Influenza-Like-Illness in the UK: Online Surveillance Using Flusurvey. BMC Infectious Diseases, 14, 232.

http://dx.doi.org/10.1186/1471-2334-14-232

[3] Molinari, N.A., Ortega-Sanchez, I.R., Messonnier, M.L., Thompson, W.W., Wortley, P.M., Weintraub, E. and Bridges, C.B. (2007) The Annual Impact of Seasonal Influenza in the US: Measuring Disease Burden and Costs. Vaccine, 25, 5086-5096. http://dx.doi.org/10.1016/j.vaccine.2007.03.046

[4] Wu, S., Metcalf, J.P. and Wu, W. (2011) Innate Immune Response to Influenza Virus. Current Opinion in Infectious Diseases, 24, 235-240. http://dx.doi.org/10.1097/QCO.0b013e328344c0e3

[5] van de Sandt, C.E., Kreijtz, J.H. and Rimmelzwaan, G.F. (2012) Evasion of Influenza A Viruses from Innate and Adaptive Immune Responses. Viruses, 4, 1438-1476. http://dx.doi.org/10.3390/v4091438

[6] Iwasaki, A. and Pillai, P.S. (2014) Innate Immunity to Influenza Virus Infection. Nature Reviews. Immunology, 14, 315-328. http://dx.doi.org/10.1038/nri3665

[7] Hurt, A.C., Hui, D.S., Hay, A. and Hayden, F.G. (2015) Overview of the 3rd Isirv-Antiviral Group ConferenceAdvances in Clinical Management. Influenza and Other Respiratory Viruses, 9, 20-31. http://dx.doi.org/10.1111/irv.12293

[8] Klepser, M.E. (2014) Socioeconomic Impact of Seasonal (Epidemic) Influenza and the Role of Over-the-Counter Medicines. Drugs, 74, 1467-1479. http://dx.doi.org/10.1007/s40265-014-0245-1

[9] Tripathi, S., White, M.R. and Hartshorn, K.L. (2015) The Amazing Innate Immune Response to Influenza A Virus Infection. Innate Immunity, 21, 73-98. http://dx.doi.org/10.1177/1753425913508992

[10] Epstein, O.I. (2008) Ultralow Doses (History of One Research). Publishing Office of the Russian Academy of Medical 
Sciences, Moscow. http://materiamedicacompany.com/en/Epstein_eng.pdf

[11] Le Goffic, R., Pothlichet, J., Vitour, D., Fujita, T., Meurs, E., Chignard, M. and Si-Tahar, M. (2007) Cutting Edge: Influenza A Virus Activates TLR3-Dependent Inflammatory and RIG-I-Dependent Antiviral Responses in Human Lung Epithelial Cells. Journal of Immunology, 178, 3368-3372. http://dx.doi.org/10.4049/jimmunol.178.6.3368

[12] Guillot, L., Le Goffic, R., Bloch, S., Escriou, N., Akira, S., Chignard, M. and Si-Tahar, M. (2005) Involvement of TollLike Receptor 3 in the Immune Response of Lung Epithelial Cells to Double-Stranded RNA and Influenza A Virus. The Journal of Biological Chemistry, 280, 5571-5580. http://dx.doi.org/10.1074/jbc.M410592200

[13] Goffic, R.L., Balloy, V., Lagranderie, M., Alexopoulou, L., Escriou, N., Flavell, R., Chignard, M. and Si-Tahar, M. (2006) Detrimental Contribution of the Toll-Like Receptor (TLR)3 to Influenza A Virus-Induced Acute Pneumonia. PLoS Pathogens, 2, e53. http://dx.doi.org/10.1371/journal.ppat.0020053

[14] Burleson, G.R. and Burleson, F.G. (2007) Influenza Virus Host Resistance Model. Methods, 41, 31-37. http://dx.doi.org/10.1016/j.ymeth.2006.09.007

[15] Heads of Medicines Agencies Homeopathic Medicinal Product Working Group (HMPWG) (2007) Points to Consider on Non-Clinical Safety of Homeopathic Medicinal Products of Botanical, Mineral and Chemical Origin. http://www.hma.eu/uploads/media/PtC_HMP_non_biological_safety_01.pdf

[16] Weber, O. (2009) The Role of Viruses in the Etiology and Pathogenesis of Common Cold. In: Eccles, R. and Weber, O., Eds., Common Cold, Birkhauser Advances in Infectious Diseases, Basel, 107-115. http://dx.doi.org/10.1007/978-3-7643-9912-2_5

[17] Hewson, C.A., Jardine, A., Edwards, M.R., Laza-Stanca, V. and Johnston, S.L. (2005) Toll-Like Receptor 3 Is Induced by and Mediates Antiviral Activity against Rhinovirus Infection of Human Bronchial Epithelial Cells. Journal of Virology, 79, 12273-12279. http://dx.doi.org/10.1128/JVI.79.19.12273-12279.2005

[18] Barrett, B., Brown, R.L., Mundt, M.P., Thomas, G.R., Barlow, S.K., Highstrom, A.D. and Bahrainian, M. (2009) Validation of a Short Form Wisconsin Upper Respiratory Symptom Survey (WURSS-21). Health and Quality of Life Outcomes, 7, 76. http://dx.doi.org/10.1186/1477-7525-7-76

[19] NIH Health Care Systems Research Collaboratory (2014) Patient-Reported Outcomes. In: Rethinking Clinical Trials: A Living Textbook of Pragmatic Clinical Trials. http://sites.duke.edu/rethinkingclinicaltrials/patient-reported-outcomes

[20] Perales-Linares, R. and Navas-Martin, S. (2013) Toll-Like Receptor 3 in Viral Pathogenesis: Friend or Foe? Immunology, 140, 153-167. http://dx.doi.org/10.1111/imm.12143

[21] Osborne, R.H., Norquist, J.M., Elsworth, G.R., Busija, L., Mehta, V., Herring, T. and Gupta, S.B. (2011) Development and Validation of the Influenza Intensity and Impact Questionnaire (FluiiQ ${ }^{\mathrm{TM}}$ ). Value in Health, 14, 687-699. http://dx.doi.org/10.1016/j.jval.2010.12.005 